tion - could be pursued in any organism by cloning and sequencing. Genetic maps, relying on breeding experiments, are required only when one wishes to operate on the organism in the real world, such as in the study of human genetic disease or in the improvement of plants and animals, but, even here, the new techniques reveal new sources of genetic variation in the form of DNA markers that greatly accelerate map construction.

In his essay entitled "A Vision of the Grail”, Walter Gilbert looks at how molecular biology in general, and the human genome project in particular, will change biology. He believes that most of the present style, if not content, of today's experimental biology will wither away, and we will purchase all the tools and information as kits and services. Sequences - even the grail sequence will be available through computer networks and the "science will have moved on to the problem of what a sequence means, what the gene actually does". He published this view last year in Nature (349, 99; 1991), and I do not know why it aroused such a violent response; true, it is a somewhat arrogant way of putting it, but after many years of hard work I, for one, look forward to an elegant life of theoretical contemplation of sequences, punctuated by the odd delightful, well-designed experiment. Of course, the lament of the older molecular biologist is that the kit revolution has already happened; most young gene hackers would not know how to go about constructing a vector or making a mutant, and even those who claim to be

discovering new human genes find them in commercial libraries.

The new genetics in fact involves a return to a much older tradition in science, that of observation and measurement, a kind of paradigm regained rather than one lost. The study of genomes differs as much from experimental biology as astronomy differs from experimental physics; 'genonomy' seems an appropriate name for this new science. For us genonomers, who chart the genetic heavens, the invention of sequencing can be compared to Galileo's invention of the telescope, and like astronomers, we can also look far back in time, reconstructing the genes and genomes of long-vanished organisms. We do not have hypotheses, and we have no experiments to perform, so we are unlikely to command the attention of those who dispense research funds on a strict Popperian basis. We do not want to stop experimental biology, because that is needed for the analysis of biological function, which tells us the meaning of our sequences. But those many hedgehogs interested in finding out everything about one thing should tolerate the few foxes who wish to find out a little about everything. In studying the human genome, much will be discovered about the evolution of life and living systems, and if, as the book tries to show, there are fears, there is also hope that this knowledge will also benefit humanity. What more can one want?

Sydney Brenner is in the MRC Molecular Genetics Unit, Hills Road, Cambridge CB2 $2 Q H$, UK.

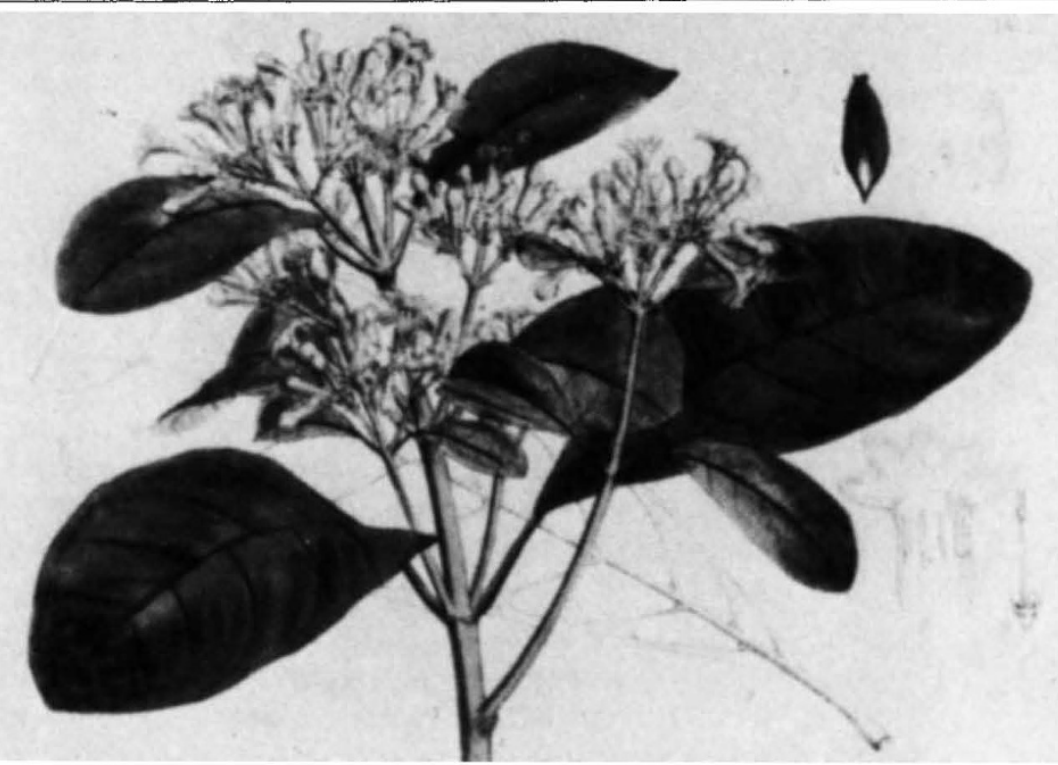

The South American tree CInchona (Rubiaceae) is widely cultivated for the dried bark of its stems and roots, the source of quinine and other antimalarial alkaloids. There are more than 10,000 species worldwide. Shown here is Cinchona ledgerina, which Is grown commercially in the tropics. The picture first appeared in Medicinal Plants by R. Bentley and H. Trimen (1880); it is now reproduced in Plants in Cardiology by A. Hollman, published by the British Medical Journal at $£ 5.95$ (pbk). 
is "Truth is what you find at the end of a correct chain of arguments."

The author quotes Einstein: "The reason why mathematics enjoys special esteem, above all other sciences, is that its laws are absolutely certain and indisputable, while those of all other sciences are to some extent debatable." Two plus two cannot be anything but four, but when one applies this tautology to the world one has to invoke what the philosopher Rudolph Carnap called "correspondence rules". These are assumptions such as that a pebble, a cow or a star is taken as 1 . If such equivalence is not assumed, then $2+2=4$ is violated by, for example, the fact that two drops of water added to two drops makes one big drop. Nothing could be simpler than this obvious distinction between pure and applied, yet some philosophers and mathematicians, frightened by the word 'truth', seem unable to grasp it.

King shares physicist Eugene Wigner's puzzlement over the "unreasonable effectiveness" of mathematics. This puzzlement puzzles me. Not only is the Universe mathematically structured, it is made entirely of mathematics. Matter consists of fields and their particles, which are not made of anything except equations. What is so mysterious about the application of these equations to a Universe from which our minds, in turn made of mathematics, originally extracted them? If mathematical patterns are not discovered, but are entirely human creations like poetry and music, then their powerful effectiveness in science and technology is indeed what Wigner called an undeserved miracle.

King is particularly fond of the poetry of Robert Frost. His poems "stay with you because of their simplicity: you come back to them for their depth . . . Frost brought poetry to the multitudes; let's now bring them mathematics." The Frosts of mathematics have yet to appear, but King is "certain as sunrise" that they will some day revolutionize mathematical teaching.

The author has a romantic vision of himself as "an aging gunfighter, walking alone the mean streets of academe". His fire is aimed at a conservative elite too self-absorbed in their specialized research to be much concerned about the public's growing mathematical illiteracy. The dull "cinder-block textbooks" get "clunkier". Although their diagrams are splattered with colour, somehow the splendour of ideas behind the diagrams remains as invisible as the textbook authors. Meanwhile the M- and N-cultures "fly apart like galaxies on opposite sides of an expanding universe".

Martin Gardner is at 110 Glenbrook Drive, Hendersonville, North Carolina 28739, USA.

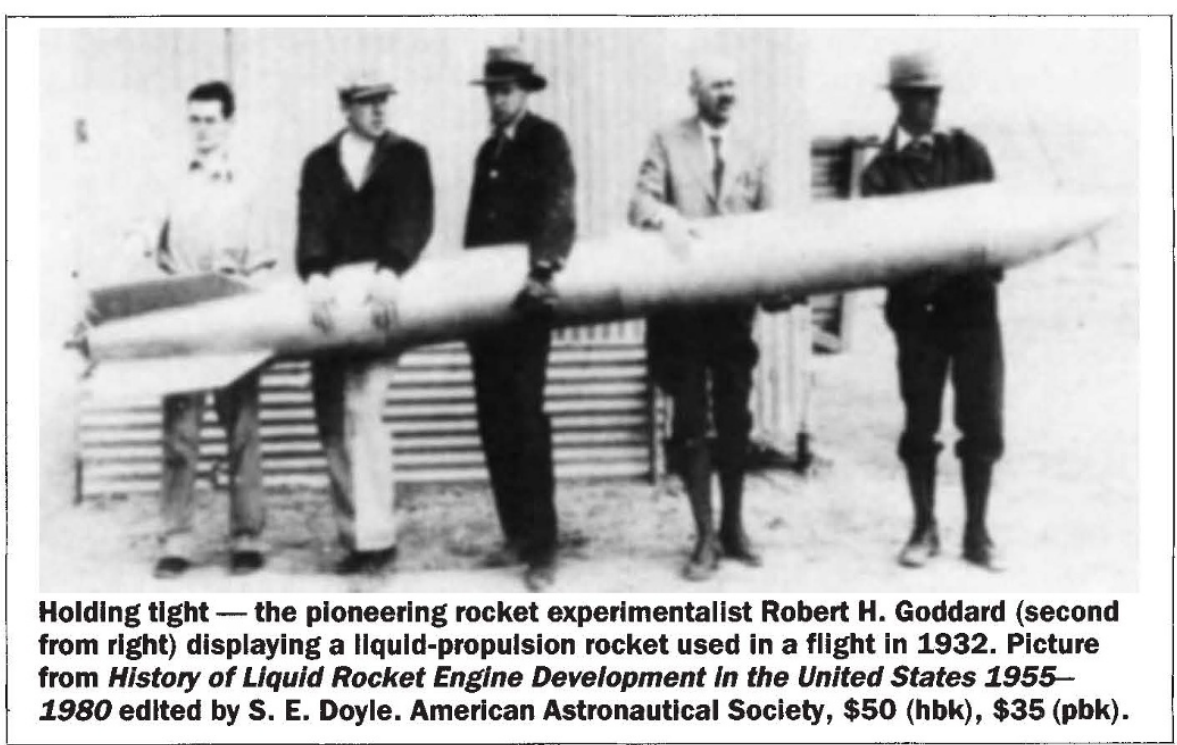

\section{Differentiating diseases}

Denis Mollison

Infectious Diseases of Humans: Dynamics and Control. By Roy M. Anderson and Robert M. May. Oxford University Press: 1991. Pp. 757. £50, \$95 (hbk); $£ 22.50, \$ 45$ (pbk).

ThroughouT history, many more people have been killed by infectious diseases than by wars. One spectacular example is the conquest of the Americas following Columbus's arrival 500 years ago. The sudden collapse of the great Aztec and Inca empires could not have happened without the help of smallpox and other 'European' diseases. Often spreading faster than the colonists themselves, these microscopic invaders in a few years reduced the native population to less than a tenth of what it had been previously.

Despite such evidence of the enormous impact of infectious diseases, it is little more than 100 years since the germ theory of disease won general acceptance through the work of Pasteur, Koch and others. Mathematical modelling of disease began in the early years of this century with, for instance, Hamer and Soper's work on the basic dynamics of outbreaks and recurrent cycles of endemic diseases such as measles. Over the past 30 years or so, the literature of theoretical epidemiology has gathered pace, rather as though it were itself a new and virulent disease.

As Anderson and May point out, much of this work has become too detached from data, as well as from public health policy. Their primary aim in this book is "to show how simple mathematical models of the transmission of infectious agents within human communities can help to interpret observed epidemiological trends, to guide the collection of data towards further understanding, and to design programmes for the control of infection and disease".

Anderson and May divide infectious diseases into two broad classes, requiring different kinds of models, according to whether the diseases are caused by microparasites or macroparasites, the key distinction being that the former reproduce directly in the human host. For each class, they begin with simple static models for a stable situation of endemic disease and with simple dynamic models, before introducing important complications such as age dependence, genetic, social and spatial heterogeneity, and the problems of control. In each case, the modelling is clearly motivated by real diseases, and the discussion tied closely to clear presentations of data across the range of human infectious diseases.

This structure works beautifully, avoiding repetition and bringing out the similarities between diseases whose behaviours are superficially very different. There is a welcome emphasis on starting with simple models, so as to avoid, in the authors' words, becoming lost in "a snowstorm of parameters". It is still insufficiently recognized in mathematical biology that the 'flexibility' of a model with many parameters often amounts to meaninglessness, in that such a model can be fitted to anything; as an eighteenth-century mathematician once put it: "give me five parameters and I will draw you an elephant, six and I will have him wave his trunk".

The authors' mathematical approach is based on differential equations. Although possibly off-putting in appearance, at least to the biological and medical scientists at whom the book is aimed, these equations are straightforward and well-understood mathematical tools, and allow the authors to consider 